\title{
Tarifliche Qualifizierungsregelungen im öffentlichen Dienst: betriebliche Umsetzung und Effekte
}

\author{
Reinhard Bahnmüller \\ Markus Hoppe
}

Vor fünf Jahren nahmen Bund, Länder und Kommunen im Rahmen der Reform ihrer Tarifverträge (TVöD/TV-L) erstmals tarifliche Regelungen zur Qualifizierung auf. Sie folgten damit dem Beispiel einiger privatwirtschaftlicher Branchen, die diesen Schritt bereits einige Jahre früher gemacht hatten. Der Beitrag informiert über den Umgang der Betriebsparteien mit den Tarifregelungen und deren Wirkungen, benennt Gründe für ihre bisher sehr schwachen Effekte und bilanziert die Möglichkeiten sowie die Grenzen von tariflichen Qualifizierungsregelungen.

\section{Zur Relevanz tariflicher Qualifizierungsregelungen und ihre Verbreitung}

Beruflicher Weiterbildung wird als Handlungsfeld gewerkschaftlicher Tarifpolitik in der wissenschaftlichen wie in der politischen Diskussion wachsende strategische Bedeutung zugesprochen. Begründet wird dies auf mehreren Ebenen: In der wissenschaftlichen Diskussion wird zum Ersten auf die Relevanz von Weiterbildung für die Verkaufsfähigkeit und den Preis der Ware Arbeitskraft hingewiesen. „Als Verkäufer von Arbeitskraft", so formuliert etwa Müller-Jentsch (1999, S. 247), „sind die Arbeitnehmer primär an der Verwertbarkeit ihrer beruflichen Fertigkeiten und Kenntnisse interessiert; diese wollen sie erhalten und steigern." Es läge deshalb auf der Hand, „dass sich auch die Sozialparteien verstärkt den Fragen der Weiterbildung und ihrer Regulierung zuwenden“ (ebd., S. 239), zumal diverse arbeitsökonomische Studien den Zusammenhang von Stabilität der Beschäftigung, Vergütung und beruflicher Weiterbildung nachweisen konnten (vgl. u.a. Backes-Gellner/Moog 2001; kritisch Wolter/Schiener 2009).

Ein zweiter Diskussionsstrang begründet den Bedarf an einer tariflichen Regulierung mit dem Kollektivgutcharakter von (Weiter-)Bildung, wodurch rational han- delnden Unternehmen Anreize geliefert würden, auf eigenes Weiterbildungsengagement zu verzichten und stattdessen zum Nutznießer der Humankapitalinvestitionen anderer Betriebe zu werden. Aufgrund dieser „Free-Rider-“ bzw. „Poaching-Problematik" bestehe die Gefahr einer Unterinvestition in Weiterbildung mit erheblichen Qualifizierungsmängeln. Dem sei nur durch tarifvertragliche oder staatliche Regulation beizukommen (Streeck 1988; Johansen 2002; Leuven 2005). Tarifverträgen wird dabei von einigen Autoren Vorrang eingeräumt, weil sie besser geeignet seien, um den unterschiedlichen Branchenbedingungen Rechnung zu tragen (Baethge et al. 2003, S. 33ff.). Zudem könne eine qualitative und um Bildungsfragen erweiterte Tarifpolitik die Heterogenität der innergewerkschaftlichen Interessen berücksichtigen, eine Antwort auf den Beschäftigungswandel darstellen sowie dem Instrument „Tarifvertrag" zu neuer Geltung und Bedeutung verhelfen (Kreft 2008, S. 110).

Schließlich wird drittens auf das Interesse der Gewerkschaften an der Erhaltung ihrer Organisationsmacht verwiesen, die sich im Kern auf die Kontrolle und Strukturierung von Arbeitsmärkten gründe. Traditionell erfolgte diese Kontrolle über das duale System der beruflichen Erstausbildung, in dem die Gewerkschaften, gestützt auf ein von gesetzlichen Regelungen flankiertes Set von Institutionen und anerkannter Kompetenz, ein einflussreicher Akteur sind. Dies gelte für die berufliche
Weiterbildung bisher nicht. Wenn sich nun die Gewichte zwischen beruflicher Erstausbildung und Weiterbildung zugunsten Letzterer verschieben, müsse es im Interesse der Gewerkschaften liegen, in der beruflichen bzw. betrieblichen Weiterbildung Fuß zu fassen, um weiterhin Einfluss auf die Arbeitsmärkte und die Verkaufsbedingungen von Arbeitskraft nehmen zu können (Bahnmüller 2009).

Auf der politischen Bühne erfährt eine tarifliche Regulierung von Weiterbildung seit geraumer Zeit ebenfalls (verhaltene) Unterstützung, die nicht zuletzt der erhofften Entlastung des Gesetzgebers geschuldet sein dürfte. Beginnend mit der rot-grünen Bundesregierung im Jahr 1998, fortgesetzt von der Großen Koalition und aktuell aufrecht erhalten von der schwarz-gelben Regierung ergingen wiederholt Appelle an die Tarifparteien, branchenbezogene Abkommen zur Förderung der betrieblichen Wei-

\footnotetext{
Reinhard Bahnmüller, Dr., Geschäftsführer des Forschungsinstituts für Arbeit, Technik und Kultur (F.A.T.K.) an der Universität Tübingen. Arbeitschwerpunkte: Industriesoziologie, Industrielle Beziehungen e-mail: reinhard.bahnmueller@unituebingen.de

Markus Hoppe, Dipl. Soz., wissenschaftlicher Mitarbeiter am F.A.T.K. an der Universität Tübingen. Arbeitsschwerpunkte: Industriesoziologie, Industrielle Beziehungen. e-mail: markus.hoppe@uni-tuebingen.de
} 
terbildung abzuschließen. Aufgeschreckt durch die Platzierung Deutschlands bei internationalen Vergleichsstudien im unteren Mittelfeld (Moraal 2009), wurde von der jetzigen Bundesregierung das Ziel formuliert, die Weiterbildungsbeteiligung in Deutschland bis 2015 auf $50 \%$ zu steigern. Die Tarifparteien wurden aufgerufen, das ihrige zur Erreichung dieses Zieles zu tun (BMBF 2008). Flankiert wird die Initiative von der Europäischen Kommission, die den Sozialpartnern ebenfalls eine „wichtige Rolle“ in der Aushandlung von Vereinbarungen zur Förderung von Weiterbildung zuweist (Kommission der Europäischen Gemeinschaft 2010, S.14).

Die Appelle blieben nicht ungehört. Die Gewerkschaften haben sich der tariflichen Regulierung von Weiterbildung im vergangenen Jahrzehnt nach langem Vorlauf verstärkt zugewandt (Busse/Seifert 2009; Bahnmüller 2009). In einigen Branchen wurden neue Tarifabkommen abgeschlossen, so etwa in der chemischen Industrie (2003), in der Metall- und Elektroindustrie (2001 und 2006), im Versicherungsgewerbe (2007) und, wovon gleich mehr zu lesen sein wird, im öffentlichen Dienst (2005/2006). Allein mit den Abkommen für den öffentlichen Dienst (ÖD) kamen ca. 2,5 Mio. Beschäftigte hinzu, deren Tarifverträge Qualifizierungsbestimmungen enthalten. Zudem gibt es mittlerweile eine erkleckliche Anzahl thematisch einschlägiger Firmentarifverträge sowie Betriebsvereinbarungen (Heidemann 2010), sodass die zur Jahrtausendwende noch zutreffende Diagnose, wonach betriebliche Weiterbildung ein weitgehend unreguliertes Feld sei (Dobischat/Seifert 2001, S.99), heute nur noch eingeschränkt zutrifft.

An Attraktivität dürfte das Thema nicht zuletzt aufgrund der verteilungspolitischen Defensive gewonnen haben, in der sich die Gewerkschaften seit den 1990er Jahren befinden. Angesichts ausbleibender Erfolge auf dem Feld der Lohnpolitik lag es nahe, nach möglichst „verteilungsneutralen“ Regulierungsfeldern Ausschau zu halten, auf denen sich tarifpolitisch gleichwohl punkten lässt. Hier bot sich u.a. die berufliche bzw. betriebliche Weiterbildung an. Zwar sind auch hier Verteilungsfragen von Zeit und Geld eingelagert, sie ließen sich jedoch ausklammern, wenn die Durchsetzung eines (quantifizierten) Rechts auf Weiterbildung für alle Beschäftigten nicht zur Maxime gemacht würde. Nicht von ungefähr erwie- sen sich dann auch solche Ansätze als Türöffner zu einer tariflichen Gestaltung von Weiterbildung, bei denen Verfahren der Bedarfsermittlung und Weiterbildungsplanung im Zentrum standen - und nicht ein Rechtsanspruch auf Weiterbildung für alle. Im Kern geht es bei diesen Abkommen um die Verpflichtung der Arbeitgeber, den Qualifizierungsbedarf des Unternehmens und der Beschäftigten zu ermitteln, beides möglichst zur Deckung zu bringen, mit dem Betriebs-/Personalrat zu beraten und darauf bezogen einen Qualifizierungsplan zu erstellen. Tarifabkommen dieses Typs gibt es für die Metall- und Elektroindustrie (M+E-Industrie), die Versicherungswirtschaft und den ÖD, der im Mittelpunkt unserer nachfolgenden Betrachtung steht.

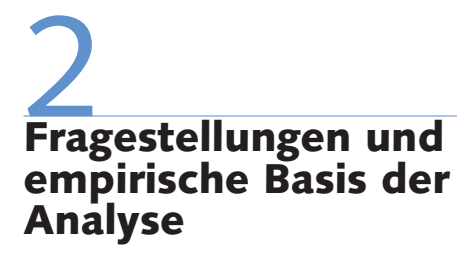

Im Zentrum unseres Interesses steht die Frage, welche Effekte die tariflichen Qualifizierungsbestimmungen für den ÖD haben und welchen Nutzen die betrieblichen Akteure in ihnen sehen. Geklärt werden soll, ob und in welcher Weise die Betriebsparteien die Vorgaben des TVöD nutzen und eigene Umsetzungsaktivitäten entwickeln. Werden Qualifizierungsgespräche, sofern noch nicht vorhanden, eingeführt? Oder wird der Personenkreis ausgeweitet, mit dem sie geführt werden? Nutzen die Beschäftigten die erweiterten Beteiligungschancen? Werden die bisher praktizierten Verfahren der Bedarfsermittlung den tariflichen Vorgaben angepasst? Und grundsätzlicher gefragt: Sind die Tarifregelungen geeignet, die betriebliche Weiterbildungspraxis im ÖD zu verbessern, und wenn ja, in welcher Hinsicht?

Für die M+E-Industrie sind die Effekte der Tarifregelung, allerdings nur für den Tarifbezirk Baden-Württemberg, bereits untersucht worden (Bahnmüller/Fischbach 2006). Eine Evaluation für andere Regionen ist über eine Vorstudie für NordrheinWestfalen nicht hinausgekommen (Lenz/ Voß 2009). Eine Übertragung der badenwürttembergischen Befunde auf andere Regionen oder gar Sektoren ist jedoch problematisch. Ihr steht die Kontextabhängigkeit der Umsetzung entgegen. Tarifliche Qua- lifizierungsbestimmungen, die, wie in der $\mathrm{M}+\mathrm{E}-$ Industrie und im ÖD, kaum materielle Mindeststandards, dafür aber Prozessvorgaben und Rahmensetzungen enthalten, müssen wahrgenommen, gedeutet und in der Umsetzung betrieblich konkretisiert werden. Ob und in welcher Weise dies erfolgen soll, lässt sich aus den Tarifregelungen selbst nicht ableiten, da sie keinen Qualitätsmaßstab einer tarifadäquaten Umsetzung mitliefern. Trotz inhaltsähnlicher Regelungen wie in der M+E-Industrie sind somit im ÖD sektorenspezifische Umgangsstrategien der betrieblichen Akteure wie auch abweichende Wirkungen zu erwarten.

Für den ÖD lassen sich mehrere Faktoren identifizieren, die einen positiven Einfluss auf die Wirksamkeit tariflicher Qualifizierungsregelungen haben könnten. Hierzu zählt die nach wie vor sehr hohe Tarifbindung von Betrieben und Beschäftigten (ca. $90 \%$ ), was der Breitenwirkung von Tarifabkommen zuträglich sein müsste (Ellguth/Kohaut 2011, S. 16). Auch hinsichtlich der Tariftreue unterscheidet sich der ÖD positiv von der Privatwirtschaft. Eine stille Tarifflucht, d.h. die Nichteinhaltung bzw. faktische Unterschreitung tariflich vereinbarter Standards, kommt Keller (2010, S. 96) zufolge „nicht vor“. Des Weiteren liegt das Qualifikationsniveau im ÖD deutlich über dem der Privatwirtschaft, was ebenfalls als weiterbildungsfördernder und damit auch umsetzungsunterstützender Faktor gelten kann. Begünstigend dürfte schließlich auch der Umstand wirken, dass den betrieblichen Akteuren mit den nunmehr verbindlich vorgeschriebenen Qualifizierungsgesprächen ein Instrument an die Hand gegeben wird, mit dem sie die in der wissenschaftlichen Diskussion vielfach konstatierten und von Personalräten bzw. Gewerkschaften häufig beklagten Kommunikations- und Führungsprobleme im ÖD angehen könnten (Naschold/ Bogumil 1998, S. 91).

Die nachfolgend präsentierten Befunde zuEffekten der Qualifizierungsbestimmungen des TVöD basieren im Wesentlichen auf einer im Sommer 2010 durchgeführten schriftlichen, standardisierten und postalischen Parallelbefragung von Personal-/ Weiterbildungsmanagern (PM) sowie Personalräten (PR) aller Kommunen ${ }^{1}$ mit mehr als 10.000 Einwohnern $(\mathrm{N}=1913)$ sowie al-

Einbezogen sind alle Städte und Gemeinden (inkl Verbandsgemeinden, Samtgemeinden und Verwaltungsgemeinschaften). 
ler Landkreise in Deutschland $(\mathrm{N}=301){ }^{2}$ Die Rücklaufquote lag bei $37 \%(n=816$; PR) bzw. $42 \%(n=927$; PM). Beantwortet wurden die Fragebögen der Personalräte zu 85 \% von den Personalratsvorsitzenden und zu $12 \%$ von deren Stellvertretern. ${ }^{3}$ Auf Arbeitgeberseite füllten sie zu $67 \%$ die Personal- bzw. Weiterbildungszuständigen aus und zu 28 \% Mitarbeiter der Personal-/ Weiterbildungsabteilung. Die Struktur des Befragungssamples der Manager sowie der Personalräte entspricht hinsichtlich der regionalen Verteilung, der Größe der Einrichtungen und der Belegschaftsstruktur weitgehend der Grundgesamtheit. Die Ergebnisse können insofern als repräsentativ betrachtet werden.

\section{Die Qualifizierungsbestim- mungen des § 5 TVöD/ TV-L: Inhalt und Ziele}

Was beinhalten die Qualifizierungsbestimmungen des $\$ 5$ TVöD/TV-L? Das Herzstück ist der Anspruch der Beschäftigten auf ein regelmäßiges, normalerweise jährlich stattfindendes Qualifizierungsgespräch mit ihrer jeweiligen Führungskraft, „in dem festgestellt wird, ob und welcher Qualifizierungsbedarf besteht“ ( $\$ 5$ Abs. 4). Dieses Gespräch kann als Einzel- oder Gruppengespräch geführt werden. Einen Anspruch auf Qualifizierungsmaßnahmen, auch solche, die in einem Qualifizierungsgespräch einvernehmlich als nötig identifiziert werden, gibt es allerdings, wie ausdrücklich betont wird, nicht.

Beschäftigte im Erziehungsdienst (im Tarifgebiet West) haben jedoch seit 2008 einen Anspruch auf 2,5 Tage bezahlte Qualifizierungs- bzw. Vorbereitungszeit pro Jahr (\$56 TVöD BT-V). Die Regelung kam im Rahmen der Kompromissbildung um die arbeitgeberseitig geforderte Arbeitszeitverlängerung zustande. Die regelmäßige wöchentliche Arbeitszeit wurde auf39 Stunden erhöht, im Gegenzug wurde Beschäftigten des Erziehungsdienstes das Recht auf Vorbereitungs- und Qualifizierungszeit im genannten Umfang eingeräumt.

Hinsichtlich der Übernahme der Kosten ist in $\$ 5$ Abs. 5 TVöD bzw. Abs. 6 TV-L geregelt, dass vom Arbeitgeber veranlasste Qualifizierungsmaßnahmen einschließlich Reisekosten grundsätzlich von ihm übernommen werden. Werden mögliche Eigenbeiträge der Beschäftigten eingefordert, sind diese in einer Qualifizierungsvereinbarung zu regeln. Dabei sind die Betriebsparteien gehalten, „die Grundsätze einer fairen Kostenverteilung unter Berücksichtigung des betrieblichen und individuellen Nutzens" zu beachten.

Betont wird das gemeinsame Interesse von Beschäftigten und Arbeitgebern an einem hohen Qualifikationsniveau und lebenslangem Lernen ( $\$ 5$ Abs. 1). Unterschieden werden vier Formen von Qualifizierungsmaßnahmen im Sinne des Tarifvertrags: Erstens solche, „die der Fortentwicklung der fachlichen, methodischen und sozialen Kompetenzen für die übertragenen Tätigkeiten dienen (Erhaltungsqualifizierung)“; zweitens jene, die geeignet sind, „zusätzliche Qualifikationen“ zu erwerben (Fort- und Weiterbildung); drittens Maßnahmen zur Arbeitsplatzsicherung (Qualifizierung für andere Tätigkeiten; Umschulung) und schließlich viertens Qualifizierungsmaßnahmen nach längerer Abwesenheit (Wiedereinstiegsqualifizierung) ( $\$ 5$ Abs. 3). Die Teilnahme an solchen Maßnahmen ist zu dokumentieren und schriftlich zu bestätigen. Beschäftigten in Teilzeitarbeit soll zudem eine gleichberechtigte Teilnahme ermöglicht werden.

Zusammenfassend und kontrastierend zu den Qualifizierungsregelungen in der $\mathrm{M}+\mathrm{E}-$ Industrie, an die sich der ÖD anlehnt, lässt sich festhalten:

(1) Primäre Stoßrichtung der Tarifregelung ist es, die Beteiligungs- und Artikulationschancen der Beschäftigten im Prozess der Weiterbildungsplanung via eines von den Beschäftigten einforderbaren Qualifizierungsgesprächs zu verbessern. Sie sollen ihre Qualifizierungsinteressen regelmäßig und institutionalisiert in die betrieblichen Planungsprozesse einbringen können. Die Qualifizierungsregelungen zielen darüber hinaus auf eine generelle Systematisierung der Bedarfsermittlungs- und Planungsprozesse in der betrieblichen Weiterbildung als Teil der Personalentwicklung.

(2) Die Qualifizierungsregelungen des TVöD/TV-L stärken die Individualrechte der Beschäftigten, nicht die Kollektivrechte der Personalräte, wobei die Individualrechte darauf beschränkt sind, den Qualifizierungsbedarf artikulieren zu dürfen. Ob sich daraus Qualifizierungsmaßnahmen ergeben, bleibt offen. Ein Anspruch auf Qualifizierung wird ausdrück- lich abgewiesen. Er besteht lediglich für Beschäftigte im Erziehungsdienst (West) im Umfang von 2,5 Tagen pro Jahr.

(3) $\$ 5$ TVöD/TV-L enthält im Gegensatz zu den Qualifizierungstarifverträgen in der M+E-Industrie keine Konfliktregelung für den Fall, dass sich die Führungskraft und der Beschäftigte nicht darauf verständigen können, ob es einen Qualifizierungsbedarf gibt.

(4) Die Möglichkeiten, sich über den Bedarf des einzelnen Unternehmens/der Verwaltung hinaus zu qualifizieren, werden nicht erweitert. Auch hierbei gehen die Regelungen für die M+E-Industrie einen Schritt weiter, indem Beschäftigten nach einer gewissen Zeit der Betriebszugehörigkeit das Recht eingeräumt wird, sich (ohne Fortzahlung der Bezüge) mit anschließender Rückkehrgarantie für Weiterbildungszwecke freistellen zu lassen.

(5) Überbetriebliche Supportstrukturen für Beratung und Unterstützung der betrieblichen Akteure, wie es sie in der M+EIndustrie Baden-Württembergs (Agentur Q) oder in der chemischen Industrie (Weiterbildungsstiftung) gibt, sind nicht vorgesehen.

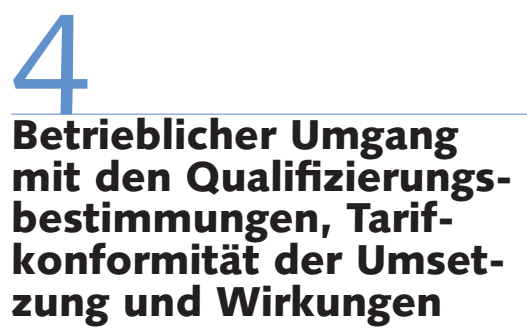

Wie sind die betrieblichen Akteure mit den Regelungen umgegangen und welche Effekte lassen sich ausmachen? Summarisch lässt sich fünf Jahre nach Inkrafttreten des

2 Die Befragung ist Teil eines vom Bundesministerium für Bildung und Forschung (BMBF) und vom Europäischen Sozialfonds geförderten Projektes „Tarifliche Regelungen zur betrieblichen Weiterbildung im öffentlichen Dienst: Implementierung, Anwendung und Wirkungen". Neben der Befragung wurden zehn Fallstudien in Kommunen, Kliniken, Hochschulen und Beratungseinrichtungen sowie zwölf Workshops mit Betriebs-/Personalräten bzw. Personal-/Weiterbildungszuständigen durchgeführt.

3 Aus Gründen der besseren Lesbarkeit wird auf die gleichzeitige Verwendung weiblicher und männlicher Sprachformen verzichtet. Gemeint und angesprochen sind immer beide Geschlechter. 
Tarifvertrags konstatieren: Die Impulse, die von den Qualifizierungsbestimmungen auf betrieblicher Ebene ausgegangen sind, waren gering. Die große Mehrheit der Kommunen und Landkreise sah in $\$ 5$ TVöD keinen Anlass für zusätzliche bzw. weiterführende Aktivitäten. Dies gilt für Personalräte wie für das Personalmanagement. Für lediglich ein knappes Viertel aller Verwaltungen war $\S 5$ ein Impuls, ihre Weiterbildungspraxis zu überprüfen. Etwa ein Fünftel der Personalräte informierte die Belegschaft über die neuen Regelungen, das Personalmanagement hielt sich dabei noch stärker zurück (11\%). Ein betriebliches Umsetzungskonzept gab es nur in $5 \%$ (PM) bzw. $11 \%$ (PR) der Verwaltungen. Zur Einrichtung oder Aktivierung einer gemeinsamen Arbeits- oder Projektgruppe zwischen Arbeitgeber und Personalrat kam es ebenfalls nur bei wenigen. Analoges gilt für Weiterbildungsausschüsse in Personalratsgremien. Neue Dienstvereinbarungen, die im Kontext des $\$ 5$ TVöD stehen, wurden in weniger als $10 \%$ der Verwaltungen abgeschlossen. Insgesamt blieben die Aktivitäten, die das Inkraftsetzen des $\$ 5$ TVöD auslöste, auf eine Minderheit von maximal einem Viertel aller Kommunen und Landkreise beschränkt (Tabelle 1).

Versucht man die Gruppe der „umsetzungsaktiven "Verwaltungen ${ }^{4}$ näher zu charakterisieren, dann ergibt sich auf der Basis einer binären logistischen Regressionsanalyse (Tabelle 2) folgendes Bild:

(1) Entgegen den in der Literatur zur Verwaltungsmodernisierung dargestellten Befunden (Bogumil et al: 2007, S. 99f; Kuhlmann/Wegrich 2001), sind die ostdeutschen Verwaltungen in Sachen $\$ 5$ TVöD erheblich umsetzungsengagierter als die westdeutschen. Der Grund hierfür ist u. E. im starken Personalabbau bei gleichzeitig notwendigem Aufbau entsprechender Qualifikationsstrukturen zu suchen. Beides verlangt ein systematisches und stringentes Vorgehen in der Personal(entwicklungs)politik.

(2) Die Größe der Verwaltungen hat bei Personalmanagern Einfluss auf die Wahrscheinlichkeit, dass sie Umsetzungsaktivitäten starten, nicht jedoch bei Personalräten. Personalmanager größerer Verwaltungen stufen ihre Einrichtung signifikant häufiger als umsetzungsaktiv ein als solche aus Verwaltungen mit weniger als 100 Beschäftigten. Bei Personalräten findet sich diesbezüglich kein Unterschied.

Tabelle 1: Betriebliche Aktivitäten bzw. Maßnahmen im Gefolge des § 5 TVöD - Angaben in \% -

Frage: War § 5 TVöD in Ihrer Verwaltung/Einrichtung Anlass für eine der nachfolgend aufgeführten Aktivitäten/Maßnahmen?*

\begin{tabular}{llccc} 
& & Trifft zu & Trifft nicht zu & Weiß nicht \\
\hline Überprüfung der betrieblichen Weiterbildungspraxis & PR & 23 & 73 & 4 \\
& PM & 22 & 75 & 3 \\
Umfassende Information der Belegschaft & PR & 21 & 77 & 2 \\
Gemeinsames Umsetzungskonzept von Personalrat & PR & 11 & 86 & 3 \\
und Personalmanagement & PM & 5 & 92 & 3 \\
Einseitiges Umsetzungskonzept von Personalrat & PR & 7 & 91 & 3 \\
oder Personalmanagement & PM & 6 & 91 & 2 \\
Einrichtung/Aktivierung einer gemeinsamen AG/ & PR & 7 & 91 & 3 \\
Projektgruppe & PM & 4 & 94 & 2 \\
Abschluss einer Dienstvereinbarung & PR & 8 & 90 & 2 \\
\end{tabular}

*(Personalräte (PR) $n=806-802$, Personal-/Weiterbildungsmanager $(P M) n=865-856$ Quelle: F.A.T.K.: ÖD-Betriebsbefragung 6/2010.

WSI MITTEILUNGEN

Tabelle 2: Einflussvariablen zur Erklärung der Wahrscheinlichkeit, Umsetzungsaktivitäten ergriffen bzw. nicht ergriffen zu haben

\begin{tabular}{|c|c|c|}
\hline Einflussfaktoren auf Umsetzungsaktivität & $\begin{array}{l}\text { Personal- } \\
\text { räte } \\
\operatorname{Exp}(B)\end{array}$ & $\begin{array}{c}\text { Personal- } \\
\text { manager } \\
\operatorname{Exp}(B)\end{array}$ \\
\hline \multicolumn{3}{|l|}{ Regionale Herkunft } \\
\hline West (Ref.: Ost) & $0,237 * * *$ & $0,325^{* * *}$ \\
\hline \multicolumn{3}{|l|}{ Betriebsgröße/Beschäftigtenzahl (Ref.: bis 100) } \\
\hline 101 bis 250 & 0,753 & 1,292 \\
\hline 251 bis 500 & 1,752 & $2,450 * *$ \\
\hline über 500 & 1,743 & $5,024^{* * *}$ \\
\hline \multicolumn{3}{|l|}{ Sensibilität und Kooperation der Akteure } \\
\hline Stellenwert von WB Führungskräfte hoch (Ref.: neutral bis niedrig) & 1,020 & 0,694 \\
\hline Weiterbildungsmotivation Beschäftigte hoch (Ref.: neutral bis niedrig) & 1,038 & 0,935 \\
\hline Kooperation zwischen PR/PM negativ (Ref.: positiv) & 0,951 & 0,833 \\
\hline Einbeziehung MA in Bedarfsermittlung (eher) gut (Ref.: neutral bis schlecht) & 0,557 & 0,647 \\
\hline Kooperation zwischen PR/PM Thema WB negativ (Ref.: positiv) & 1,078 & 0,455 \\
\hline \multicolumn{3}{|l|}{ Strukturelle Gegebenheiten der PE und WB und deren Bewertung } \\
\hline Angebot unter Bedarf (Ref.: mindestens bedarfsdeckend) & $2,315^{* * *}$ & 1,589 \\
\hline Umfang Angebot (eher) gut (Ref.: neutral bis schlecht) & 0,890 & 1,147 \\
\hline Qualität Angebot (eher) gut (Ref.: neutral bis schlecht) & 1,130 & 1,547 \\
\hline Finanzielle Aufwendungen (eher) gut (Ref.: teils teils bis schlecht) & $0,579 *$ & 0,925 \\
\hline Personal für Personalentwicklung nicht vorhanden (Ref.: vorhanden) & 1,083 & 1,378 \\
\hline Personal für Weiterbildung nicht vorhanden (Ref.: vorhanden) & 0,925 & 0,819 \\
\hline Fortbildungsprogramm nicht vorhanden (Ref.: vorhanden) & 0,749 & 1,030 \\
\hline Systematische Weiterbildungsplanung nicht vorhanden (Ref.: vorhanden) & 0,771 & $0,468 * * *$ \\
\hline Systematik/Qualität der Planung (eher) gut (Ref.: neutral bis schlecht) & 1,639 & 0,821 \\
\hline Referenzkategorie (jeweils angegebene Vergleichsgruppe) & 1 & 1 \\
\hline Konstante & $-1,073 * * *$ & $-1,895 * * *$ \\
\hline Modellgüte (Nagelkerkes R-Quadrat) & 0,189 & 0,187 \\
\hline \multicolumn{3}{|l|}{$\begin{array}{l}\text { Das Modell basiert auf einer binären logistischen Regressionsanalyse mit umsetzungsaktiv/ } \\
\text { nicht umsetzungsaktiv als abhängiger Variable. }{ }^{* * * / * * / *} \text { signalisieren einen signifikanten } \\
\text { Unterschied auf dem } 1 \%-/ 5 \%-/ 10 \%-\text { Niveau. }\end{array}$} \\
\hline Quelle: F.A.T.K.: ÖD-Betriebsbefragung 6/2010. & & \\
\hline
\end{tabular}

(3) Bei den strukturellen Gegebenheiten der Personalentwicklung und Weiterbildung sind von den geprüften Variablen im Schätzmodell bei den Personalräten nur zwei und bei den Personalmanagern eine einschlägig. Aktivitäts- bzw. umsetzungs-
4 Als "umsetzungsaktiv“ wurden jene Verwaltungen eingestuft, die Gespräche bzw. Verhandlungen zwischen Personalrat und Management im Gefolge des § 5 TVöD aufnahmen und nicht angaben: „§ 5 war kein Thema“ bzw. "§ 5 wurde diskutiert, aber kein Handlungsbedarf gesehen, da die bestehende Praxis tarifliche Ansprüche bereits abdeckt." 
fördernd ist bei Personalräten ein unter dem Bedarf liegendes Angebot, aktivitätsmindernd sind (eher) gut bewertete finanzielle Aufwendungen für Weiterbildung. Bei Personalmanagern wirkt das Fehlen einer systematischen Weitebildungsplanung umsetzungsmindernd, d.h. Umsetzungsaktivität gilt in dieser Akteursgruppe als positiver Verstärker der eigenen Professionalität.

(4) Keinen signifikanten Einfluss haben bemerkenswerter Weise bei beiden Akteursgruppen alle Variablen, welche die Sensibilität und Kooperation der betrieblichen Akteure erfassen: die Zusammenarbeit zwischen Personalrat und Management, der Stellenwert, den Führungskräfte der Weiterbildung zumessen, die Weiterbildungsmotivation der Beschäftigten und deren Einbeziehung in die Weiterbildungsplanung.

Was bedeutet nun der Umstand, dass die meisten Verwaltungen keine Umsetzungsaktivitäten ergriffen haben, für die Tarifkonformität? Lässt sich daraus automatisch schließen, sie verhielten sich nicht tarifkonform? Diese Schlussfolgerung wäre vorschnell, kann die Tarifbestimmung doch auch deshalb für „erledigt“ erklärt werden, weil die dort vorgegebenen Mindestanforderungen bereits vor Inkrafttreten der Tarifbestimmungen erfüllt wurden. Mit dieser Begründung hat auch ein Gutteil der Verwaltungen reagiert und $₫ 5$ TVöD quasi ad acta gelegt. Zu Recht? Die Frage ist nicht leicht zu beantworten, da dieser Paragraf außer dem Anspruch der Beschäftigten auf ein Qualifizierungsgespräch sowie der Regelung, wonach vom Arbeitgeber veranlasste Qualifizierungsmaßnahmen auch von ihm zu bezahlen sind, keine weiteren materiellen Vorgaben enthält, insbesondere keine, welche die Qualifizierungsgespräche betreffen. Es sind, wie bereits dargestellt, lediglich Inhalt und Ziel des Gesprächs vorgegeben (Klärung des Qualifizierungsbedarfs), nicht jedoch Form und Qualität. Qualifizierungsgespräche im Sinne des Tarifvertrags können somit viele Formen annehmen und eine sehr unterschiedliche Qualität haben. Ziehen wir dies in Betracht und legen wir ein breiteres Verständnis von Qualifizierungsgesprächen zugrunde, lässt sich zur Realisierung des Tarifanspruchs Folgendes sagen:

(1) Qualifizierungsgespräche, Mitarbeitergespräche oder vergleichbare Formen institutionalisierter Mitarbeiterkommu- nikation werden in $59 \%$ (PM) bzw. $56 \%$ (PR) aller Verwaltungen geführt. ${ }^{5}$ Eigenständige Qualifizierungs- oder Personalentwicklungsgespräche sind selten (PM 4 \%, PR $3 \%$ ). Die vorherrschende Form sind Mitarbeitergespräche, in deren Rahmen ein Gesprächsmodul „Qualifizierung“ bzw. „Personalentwicklung“ integriert ist oder sein kann (53\% PR, 55 \% PM).

(2) In den meisten Verwaltungen, die Qualifizierungsgespräche - in welcher Form auch immer - anbieten, werden sie nur mit Teilen der Belegschaft geführt. Nach Angaben der Manager liegt die durchschnittliche Durchführungsquote bei $59 \%$, die Personalräte setzen sie bei $48 \%$ an. Zudem werden die Gespräche in etwa einem Drittel der Fälle auch nicht regelmäßig, sondern nur gelegentlich bzw. bei Bedarf geführt. ${ }^{6}$

Für die Frage der Tarifkonformität lässt sich daraus folgern: Qualifizierungsgespräche werden in knapp der Hälfte der Verwaltungen nicht angeboten (PR $44 \%$, PM 41 \%). Auch dort, wo grundsätzlich ein entsprechendes Angebot existiert, ist nur etwa die Hälfte der Beschäftigten einbezogen. Nimmt man beides zusammen, ist zu konstatieren, dass etwa $70 \%$ der Beschäftigten kein Qualifizierungsgespräch führen, sei es, weil es ihnen nicht angeboten wird, sei es, dass sie es nicht einfordern. Von weitgehend tarifkonformem Verhalten lässt sich demnach kaum sprechen. Aus der Perspektive der befragten Manager und auch der Personalräte stellt sich dies allerdings anders dar. Danach gefragt, ob die betriebliche Weiterbildungspraxis in ihrer Einrichtung den Regelungen des $₫ 5$ TVöD entspricht, gibt nur eine Minderheit von 20 \% (PR) bzw. 3 \% (PM) an, die Anforderungen des Tarifvertrags würden in ihrer Verwaltung nicht erfüllt. Wörtlich voll erfüllt sieht sie der Großteil der Befragten allerdings auch nicht (PR $6 \%$, PM $14 \%)$. Die Mehrheit bewegt sich in einem Mittelfeld, hält die Vorgaben zwar nicht für wörtlich, aber für sinngemäß oder teilweise erfüllt (PR $64 \%$; PM 78 \%).

Wirkungen konnte $₫ 5$ TVöD nur dort entfalten, wo die Betriebsparteien den von den Tarifparteien gegeben Impuls tatsächlich aufgriffen, Gespräche zur Umsetzung geführt und Veränderungen angegangen wurden. Aber auch in dieser Gruppe der „umsetzungsaktiven“ Verwaltungen, die wir oben charakterisiert haben und die wir auf maximal ein Viertel aller Verwaltungen taxieren, registrieren die Befragten nicht durchweg Wirkungen. Der Anteil liegt bei etwa einem Fünftel. Gruppiert man die Wirkungsebenen nach inhaltlichen Feldern, ergibt sich folgendes Bild: In $18 \%$ (PR) bzw. $20 \%$ (PM) der Verwaltungen wurden, angestoßen durch $\S 5$, erstmals Qualifizierungsgespräche durchgeführt, in $13 \%$ (PR) bzw. $20 \%$ (PM) wurde der Personenkreis, mit dem sie geführt werden, ausgeweitet. Instrumente der Bedarfsermittlung wurden in $10 \%$ (PR) bzw. $12 \%$ (PM) der Fälle neu entwickelt oder optimiert, in $9 \%$ (PR) bzw. $17 \%$ (PM) wird eine Systematisierung der Bedarfsermittlung konstatiert. Vergleichbar häufig wird von größerer Transparenz im Weiterbildungsgeschehen und von verbesserter Dokumentation und Zertifizierung berichtet. Selten kam es zu der von Gewerkschaft und Personalräten befürchteten Kostenverlagerung auf Beschäftigte (PR/PM $8 \%$ ). Bezieht man die Angaben der Befragten zu den Wirkungen von $\$ 5$ nicht nur auf die umsetzungsaktiven Verwaltungen, sondern auf alle, reduziert sich der Anteil der Befragten, der Wirkungen konstatiert, erheblich. Je nach abgefragter Wirkungsebene liegt er zwischen $2 \%$ und $8 \%$. Die schwachen Effekte drücken sich auch in der Gesamtbewertung des $\$ 5$ TVöD aus. Die große Mehrheit hält ihn für gut gemeint, billigt ihm jedoch wenig Einfluss auf die betriebliche Weiterbildungspraxis zu (Tabelle 3).

\section{Ursachen der schwachen Impulswirkung}

Woran liegt es, dass die Tarifregelungen bisher wenig beachtet wurden und schon deshalb nur schwache Effekte hatten? Unseres Erachtens sind hierfür vor allem folgende Gründe maßgeblich:

5 Die Werte liegen leicht unter den von Bogumil et al. (2007, S. 61) im Jahr 2006 ermittelten $62 \%$. Eine Ausweitung der Mitarbeitergespräche hat, wie sich daraus folgern lässt, seither offensichtlich nicht stattgefunden.

6 An Gesprächen im Kontext leistungsorientierter Bezahlung nach § 18 TVöD (LOB-Gespräche) nehmen deutlich mehr Beschäftigte teil. Geführt werden sie in $72 \%$ (PM) bzw. $69 \%$ (PR) aller Verwaltungen, und dort beteiligen sich durchschnittlich $89 \%$ (PM) bzw. $85 \%$ (PR) aller Beschäftigten. 
(1) Charakter der Tarifregelung: Ein erster Grund dürfte im Charakter der Tarifregelung zu suchen sein. Die Regelungen sind primär prozessualer Natur und enthalten nur wenige materielle Mindestnormen, die verbindlich einzuhalten sind. Darin liegt zwar einerseits der Charme eines solchen Regulierungsansatzes, da betriebsspezifische und dem unterschiedlichen Qualifizierungsbedarf angepasste Lösungen gefunden werden können, zugleich jedoch auch seine Schwäche. Er ist darauf angewiesen, dass die Betriebsparteien den Impuls der Tarifparteien aufgreifen. Tun sie dies, d.h. werden Qualifizierungsgespräche gut vorbereitet, in qualifizierter Weise geführt und sorgfältig nachbearbeitet, wird den Betrieben/Verwaltungen wie den Beschäftigten einiges abverlangt (Neuberger 2008; Breisig 2001; ver.di 2011). Die Betriebsparteien müssen allerdings nicht handeln, sie können. Eine verpflichtend abzuschließende Dienstvereinbarung zu $\$ 5$ TVöD ist nicht vorgesehen. Auch sind keine inhaltlichen Mindestanforderungen vereinbart, aus denen sich ableiten ließe, auf welcher Basis Qualifizierungsgespräche geführt und wie sie nachbereitet werden müssen. Man kann den Weiterbildungsbedarf gründlich oder oberflächlich ermitteln, einen Qualifizierungsbedarf feststellen und die Maßnahmen hernach nicht umsetzen, eine Dienstvereinbarung zur Qualifizierung abschließen oder auch nicht. Tarifkompatibel in formalem Sinne kann vieles sein. Es ist diese „Zahnlosigkeit“, die es leicht macht, die Regelung nicht aufzugreifen, weil unter Bezugnahme auf den Tarifvertrag keine Möglichkeit eröffnet wird, für notwendig erachtete Veränderungen in Gang zu setzen und auf Qualität zu pochen.

(2) Geringer Problem- und Handlungsdruck, Dominanz anderer Themen: Eine zweite Ursache dürfte in dem nur bedingt vorhandenen Problem- und Handlungsdruck der Betriebsparteien zu suchen sein. Weiterbildung ist aus Sicht der Mehrheit der Personalräte kein Handlungsfeld mit gravierenden Defiziten. Es gibt Kritik, sie wird jedoch zumeist nicht vehement vorgetragen. So werden z.B. die Qualität der Bedarfsermittlung und die Weiterbildungsplanung von den Personalräten deutlich kritischer gesehen als von den Personal-/ Weiterbildungsverantwortlichen. Auch werden die Möglichkeiten der beruflichen Entwicklung oder die Einbeziehung der

Tabelle 3: Gesamtbewertung des § 5 TVöD - Angaben in \% -

Frage: Wie bewerten Sie den § 5 TVöD insgesamt? Bitte geben Sie an, welcher der nachfolgenden Positionen Sie am ehesten zustimmen. *

\begin{tabular}{|c|c|c|}
\hline & PR & PM \\
\hline Die Regelung ist unnötig und wirkt eher behindernd als fördernd. & 2 & 3 \\
\hline $\begin{array}{l}\text { Die Regelung ist gut gemeint, hat aber wenig Einfluss auf die betriebliche } \\
\text { Weiterbildungspraxis. }\end{array}$ & 79 & 79 \\
\hline Die Regelung ist eine gute Sache, sie hat die betriebliche Weiterbildung verbessert. & 16 & 18 \\
\hline $\begin{array}{l}\text { Die Regelung ist eine sehr gute Sache, sie hat die betriebliche Weiterbildungspraxis } \\
\text { deutlich verbessert. }\end{array}$ & 1 & 0 \\
\hline
\end{tabular}

Beschäftigten in die Bedarfsermittlung von einem Großteil bemängelt. Im Großen und Ganzen zeigen sich die Personalräte jedoch zufrieden, etwa mit dem Umfang und der Qualität des Angebots oder mit der Praxis der Kostenübernahme, die eine Mehrheit von $53 \%$ bzw. $58 \%$,gut“ oder ,eher gut“ bewerten. Nur eine Minderheit der Personalräte bemängelt, das Weiterbildungsangebot liege erheblich unter dem Bedarf (14\%), um die Chancengleichheit zur Teilnahme an Weiterbildung sei es schlecht bestellt (9\%) oder die Organisation und Zuständigkeiten für Weiterbildung böten Anlass, sie (eher) schlecht zu bewerten (32\%). Einen Grund, sich mit Nachdruck für eine Verbesserung der Weiterbildungssituation einzusetzen, sieht eine Mehrheit derzeit offensichtlich nicht. Das wird am Stellenwert des Themas in der Arbeit der Personalratsgremien deutlich. Dieser ist nach Selbsteinschätzung in $41 \%$ der Personalratsgremien (eher) gering, $28 \%$ stufen ihn als „mittel“ und lediglich $31 \%$ als (eher) hoch ein. ${ }^{7}$ In einer Rangordnung, gebildet aus neun Arbeits- bzw. Politikfeldern des Personalrats, rangiert Qualifizierung/ Weiterbildung auf dem vorletzten Platz, auf dem letzten liegt das Thema „demografischer Wandel“". Es zeigt sich somit die Kehrseite des überdurchschnittlich hohen Qualifikationsniveaus der Beschäftigten, dem wir eine eher förderliche Wirkung für den Umgang mit dem $₫ 5$ TVöD zugesprochen hatten: die Weiterbildungsbeteiligung, der Umfang und die Qualität des Angebots sowie die Chancengleichheit sind nach Meinung der meisten Personalmanager und auch der Personalräte auf einem ansprechenden Entwicklungsstand. Es wird zwar das eine oder andere bemängelt, Grund für eine Weiterbildungsinitiative anlässlich der Umsetzung von $\$ 5$ wird jedoch zumeist nicht gesehen.

Es gab und gibt andere Themen, die wichtiger erscheinen und denen Vorrang eingeräumt wird. Eines beschäftigte die betrieblichen Akteure ganz besonders: die leistungsorientierte Bezahlung (LOB) (Schmidt et al. 2011a). In der Selbsteinschätzung der Personalräte liegt deren Stellenwert in der Gremienarbeit deutlich über jenem der Weiterbildung. Von den Arbeitgebern gefordert und zur Bedingung für den Abschluss des TVöD erklärt, von der Gewerkschaft ver.di nur widerwillig akzeptiert, verdrängte die Debatte um die Gestaltung des Leistungsentgelts das Qualifizierungsthema weitgehend (Sterkel et al. 2010). Mit dazu beigetragen hat die auch strategisch gewollte Separierung der Politik- und Gestaltungsfelder Leistungsentlohnung einerseits und Qualifizierung andererseits. Ver.di war darauf bedacht, die (ungewollte) leistungsorientierte Bezahlung von den (gewollten) Qualifizierungsregelungen möglichst fern zu halten (ver.di 2008). Die Akzeptanz von LOBGesprächen sollte nicht dadurch gefördert werden, dass sie mit Qualifizierungsgesprächen kombiniert oder beide gar integriert werden. Wo die Etablierung von Qualifizierungsgesprächen nach $\$ 5 \mathrm{TVöD}$ auf der Agenda blieb, wurden - meist aufgrund der Ressourcenprobleme der Führungskräfte - die Weichen gleichwohl von Beginn an auf „Integration“ gestellt. Oder es blieb den Führungskräften sowie den Beschäftigten überlassen, ob sie die LOBGespräche und die Qualifizierungsgespräche zusammen oder getrennt führen wollten. Die Mehrzahl entschied sich für eine Integration.

\footnotetext{
7 Der nachrangige Stellenwert von Weiterbildung im Betriebsratshandeln bzw. die Diskrepanz zwischen den mitbestimmungsrechtlichen Möglichkeiten und deren (geringer) Nutzung wurden schon häufig diagnostiziert (Heimann 1992, S. 325; Grass 1997, S. 157f.; Dobischat/Seifert 2001, S. 97). Eine nachhaltige Veränderung in den Prioritäten scheint es bislang nicht gegeben zu haben.
} 
(3) Eingeschränkte Akzeptanz von Qualifizierungsgesprächen und verbreite „Exit-Kultur“: Ein dritter Grund ist die eingeschränkte Akzeptanz von Mitarbeitergesprächen, die zu den „Klassikern“ der Personalentwicklung gehören und in aller Regel als „Träger“ des Modus „Qualifizierungsgespräch “ fungieren. Trotz ihres Stellenwerts in der Personalentwicklung werden Mitarbeitergespräche häufig nicht durchgeführt. Widerstände gibt es bei Führungskräften wie bei Beschäftigten. Ähnliches gilt für Qualifizierungsgespräche. Der Anspruch auf ein Qualifizierungsgespräch wird, wie unsere Befunde zeigen, vielfach auch durch die Beschäftigten nicht eingelöst. Verschärft wird das Akzeptanzproblem dadurch, dass Qualifizierungs- oder Mitarbeitergespräche wie andere PE-Instrumente häufig mit einer Exit-Option versehen sind. Es besteht fast durchweg die Möglichkeit, sich der Teilnahme zu entziehen. Die Exit-Optionen sind meist durch Interventionen der Personalvertretungen zustande gekommen, die mit Verweis auf den Schutz der Beschäftigten den Verpflichtungscharakter von PersonalEntwicklungs (PE)-Instrumenten herabzusetzen versuchen - eine aus unserer Sicht durchaus diskussionswürdige Deutung von Schutzbedürfnissen, zumal gerade bei Personalräten lautstark Klage über eine unterentwickelte Gesprächs- und Führungskultur geführt wird. Jedenfalls dürfte diese „Kultur der Freiwilligkeit“, wo vieles möglich, aber wenig verpflichtend ist, mit dazu beitragen, dass bei vorhandenen PEInstrumenten Verbindlichkeit nicht hergestellt wird und gewünschte Wirkungen verpuffen.

\section{(4) Entstehungsgeschichte der Tarifrege-} lung: Ein Gutteil der Umsetzungsprobleme dürfte sich auch aus der Entstehungsgeschichte der Tarifregelungen erklären. Sie sind kein Ergebnis einer speziell auf die Qualifizierungsthematik zugespitzten Tarifbewegung, auf welche die Mitglieder und Funktionäre durch eine breite Informationskampagne vorbereitet worden sind, für die mobilisiert und betrieblich wie überbetrieblich gestritten wurde. Vielmehr sind sie Teil des komplexen, eine Vielfalt von Themen umfassenden und schiedlichfriedlich auf dem Verhandlungswege verabschiedeten Reformprojektes TVöD (Meerkamp 2008; Schmidt et al. 2011b). Der $\$ 5$ war in dem komplexen Reformpaket nur ein Abschnitt und sicher nicht der wichtigste. Dass Forderungen zur Qualifizierung in das Forderungspaket aufgenommen wurden, war den mit dem Reformprojekt näher Vertrauten sicher bekannt, gleichwohl blieb es eine Spezialistendiskussion strategisch denkender Funktionäre und Tarifpolitiker, die wenig Breitenwirkung hatte. Es bestätigt sich die auch in anderen Branchen gemachte Erfahrung: Tarifforderungen zur Weiterbildung, für die nicht im Rahmen einer Tarifbewegung geworben und mobilisiert wurde, finden betrieblich wenig Resonanz und lassen sich deshalb schwer umsetzen (Bahnmüller et al. 1993; Seitz 1997; Lenz/Voß 2009).

(5) Umsetzungsbegleitung durch die Tarifparteien: Hinzu kommt, dass die Umsetzung der im Tarifvertrag vereinbarten Qualifizierungsregelungen in den Betrieben/Verwaltungen durch die Tarifparteien kaum begleitet worden ist. Die Vereinigung der kommunalen Arbeitgeberverbände (VKA) sieht es - mit Differenzierungen bei einzelnen Themenfeldern - nicht als ihre Aufgabe an, ihre Mitglieder (von juristischem Rat abgesehen) in der Umsetzung tariflicher Regelungen zu unterstützen, um eine ihren Intentionen entsprechende Auslegung und Ausgestaltung sicherzustellen. Auch seitens der Gewerkschaft ver.di fand eine Umsetzungsbegleitung nur sehr eingeschränkt statt. Dies lag allerdings weniger daran, dass ver.di entsprechende Aktivitäten als Aufgabe zurückweisen würde. Es fehlte vielmehr an entsprechender Erfahrung. Umsetzungsstrukturen und eine entwickelte Umsetzungskultur von Tarifverträgen existieren bisher kaum. Sie waren bislang auch nicht nötig, da die Umsetzung von Tarifverträgen meist über Verwaltungsakte erfolgte (Dienstanweisung, gesetzliche Regelung). Eine Umsetzungsund Betriebspolitik, vergleichbar jener in der Privatwirtschaft, war somit nicht erforderlich. Dies ändert sich jedoch in dem $\mathrm{Maße}$, in dem die Dezentralisierung der Tarifpolitik voranschreitet und vermehrt qualitative sektorale Rahmenabkommen mit betrieblichem Konkretisierungsbedarf abgeschlossen werden (Bahnmüller 2010; Keller 2010, S. 90ff.). Die Regelungen des $\$ 5$ sind ein Beispiel dafür, jene zum Leistungsentgelt ( $\$ 18$ TVöD) oder zur Arbeitszeitgestaltung sind andere. Daraus ergeben sich neue Aufgaben für die Gewerkschaft, vor allem die Tarifpolitik und gewerkschaftliche Betriebspolitik als zwei Seiten einer Medaille zu betrachten. Sollen gestaltungsoffene Tarifverträge entspre- chend gewerkschaftlichen Intentionen im Betrieb umgesetzt werden, muss gewerkschaftliche Betriebspolitik einen höheren Stellenwert erhalten und nach dem Motto verfahren werden: Keine qualitative Tarifregelung ohne gewerkschaftliches Umsetzungskonzept. Im Fall des $₫ 5$ TVöD lag ein solches nicht vor, was nicht ohne Folgen für das Ergebnis blieb.

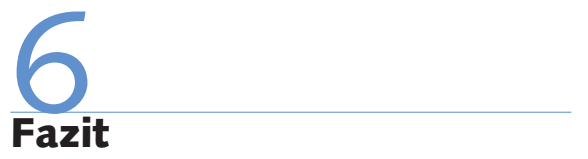

Zieht man eine vorläufige Bilanz der Umsetzung, ist das Ergebnis ernüchternd. Die Wirkungen sind schwach, schwächer noch als in der M+E-Industrie. Die These, wonach im ÖD die Tarifregelungen aufgrund des überdurchschnittlich hohen Qualifikationsniveaus der Beschäftigten, der vielfach beklagten Kommunikationsund Führungsdefizite und dem (bisher) tariftreuen Verhalten der Verwaltungen auf fruchtbareren Boden als in der M+EIndustrie fallen würden, muss verworfen werden. Das Gegenteil ist der Fall: Umsetzungsaktivitäten der Betriebsparteien sind seltener, die Effekte sind auf einen kleineren Kreis von Betrieben/Verwaltungen begrenzt und sie sind deutlich schwächer. Zudem werden Qualifizierungsregelungen von den Betriebsparteien im ÖD, insbesondere von den Personalräten, kritischer bewertet als in der M+E-Industrie (Bahnmüller/Fischbach 2006, S. 211).

Mit der Umsetzung dieses Typs tarifvertraglicher Qualifizierungsregelungen tun sich allerdings alle schwer. Auch in der M+E-Industrie sind die Wirkungen der Qualifizierungstarifverträge überschaubar (Bahnmüller/Fischbach 2006, S. 198ff.). Zwischen den realen Effekten der Qualifizierungsabkommen und der strategischen Bedeutung, die dem Feld zugeschrieben wird, besteht somit eine beträchtliche Lücke. Lässt sie sich mit einem Ansatz, wie er im ÖD, der M+E-Industrie und seit Kurzem auch in der Versicherungswirtschaft verfolgt wird, wirklich schließen? Lassen sich auf diesem Weg die von den Gewerkschaften beklagten Defizite abbauen, die Gefahr einer systematischen Unterinvestition in Humankapital bannen, die Weiterbildungsbeteiligung steigern, die soziale Selektivität reduzieren, die Bedarfsermittlung und Weiterbildungsplanung nachhaltig verbessern? 
Legt man diese Messlatte an, fällt die Antwort - mit Differenzierungen bei einzelnen Zielgrößen - eher negativ aus. Für eine Erhöhung der Weiterbildungsteilnahme aufgrund tariflicher Qualifizierungsregelungen, eine Verringerung der sozialen Selektivität oder eine Steigerung der Investition in Weiterbildung gibt es keine bzw. nur schwache Belege (Düll/ Bellmann 1998; Seitz 1997; Bahnmüller/ Fischbach 2006). Allerdings ist der im ÖD wie in der M+E-Industrie gewählte tarifpolitische Ansatz auch nicht darauf ausgerichtet, auf diesen Ebenen wirksam zu sein. Sie werden, wenn überhaupt, nur mittelbar tangiert. Insofern darf $\$ 5 \mathrm{TV}$ ÖD wie die vergleichbaren Regelungen in der $\mathrm{M}+\mathrm{E}-$ Industrie oder im Versicherungsgewerbe an solchen Ansprüchen auch nicht gemessen werden. Die Erwartungen sollten dem tarifpolitischen Ansatz und seinen Potenzialen angemessen sein. Diese zielen im Falle der hier diskutierten Regelungen auf einen anderen Aspekt, nämlich primär auf die Chancen der Beschäftigten, ihre Interessen in die Bedarfsermittlung bei der Qualifizierungsplanung institutionalisiert einbringen zu können. Und darauf bezogen kann festgestellt werden: Der Anspruch auf ein Qualifizierungsgespräch existiert, die Beteiligungschancen sind ausgeweitet. Sie werden allerdings nur bedingt genutzt bzw. offensiv eingefordert. Es gibt offensichtlich verbreitet Zweifel am Nutzen der Gespräche. Und solche Zweifel sind oftmals auch nicht unbegründet. Weshalb sollen Qualifizierungsgespräche geführt und der Qualifizierungsbedarf festgestellt werden, wenn die für Qualifizierungen notwendigen finanziellen Mittel absehbar nicht oder nur unzureichend verfügbar sind? Weshalb soll jährlich über die beruflichen Entwicklungsmöglichkeiten gesprochen werden, wenn diese in der Einrichtung ausgesprochen limitiert oder schlicht nicht vorhanden sind? Zweifel am Nutzen der Qualifizierungsgespräche machen sich nicht zuletzt an solchen Diskrepanzen und damit an der tatsächlichen oder vermeintlichen Folgenlosigkeit der Gespräche fest, wobei zur Frustration mit beiträgt, wenn der Nutzen der Gespräche primär an der Aussicht auf eine besser dotierte Stelle bemessen wird.

Der $₫ 5$ TVöD wie die vergleichbaren Tarifregelungen in anderen Branchen waren die Türöffner für eine tarifliche Regulierung von Weiterbildung. Sie sollten für die Gewerkschaften ein Einstieg sein - und dieser ist erfolgt. Allerdings kann es dabei nicht bleiben, wenn die Gewerkschaften ihren Anspruch einlösen wollen, sich und die Betriebs-/Personalräte als einflussreiche Akteure in der beruflichen Weiterbildung zu etablieren sowie substanzielle Verbesserungen auch im Hinblick auf Finanzierung, Weiterbildungsteilnahme und soziale Selektivität zu erreichen. Hierzu reichen Tarifregelungen wie der $\$ 5$ TVöD offensichtlich nicht aus. Die Wirksamkeit dieses Typs von Abkommen könnte allerdings gesteigert werden, wenn erstens im Vorfeld eines solchen Tarifabschlusses eine gründliche Analyse der branchenspezifischen Bedingungen und Defizite in der Weiterbildung vorgenommen würde. Zum zweiten wäre sicherzustellen, dass bei den betrieblichen Akteuren, allen voran bei den Betriebs-/Personalräten, Akzeptanz und Anwendungsbereitschaft für die beschlossenen bzw. ins Auge gefassten Lösungen vorhanden sind. Zum Dritten dürften ta- rifliche Qualifizierungsregelungen nicht beiläufig und lautlos vereinbart werden, sondern sie sollten als Ergebnis einer Tarifbewegung von intensiven betrieblichen Debatten begleitet sein. Notwendig wäre schließlich viertens ein besserer externer Support der Betriebsparteien im Prozess der Umsetzung qualitativer tariflicher Regelungen. Bedarf hierfür haben nicht nur, aber vor allem Personalräte. Ihre zeitlichen Ressourcen und fachlichen Kompetenzen scheinen oftmals nicht hinreichend, den sich vielfach über Jahre hinstreckenden Umsetzungsprozess ausschließlich mit eigenen Kapazitäten zu bewältigen. Was an Unterstützung von außen notwendig ist, sollte bereits bei der Entwicklung der Tarifforderungen mitbedacht werden und kann auch Gegenstand des Verhandlungsprozesses bzw. des Tarifabschlusses sein, wie die von beiden Tarifparteien getragene Agentur Q in der baden-württembergischen $\mathrm{M}+\mathrm{E}-$-Industrie oder die „Stiftung Weiterbildung" in der chemischen Industrie zeigen. Die Tarifparteien sollten jedenfalls aus der Verantwortung auch für die Umsetzung der von ihnen abgeschlossenen Verträge nicht entlassen werden.

Bei allen Schwächen in der Vorbereitung und Umsetzungsbegleitung ist jedoch auch nicht aus dem Auge zu verlieren, dass man, wie die Gruppe der umsetzungsaktiven Verwaltungen zeigt, Tarifregelungen wie den $\S 5$ TVöD durchaus zum Anlass nehmen kann, die Weiterbildungspraxis auf den Prüfstand zu stellen und Qualifizierungsgespräche zu einem wichtigen Teil eines umfassenden Personalentwicklungskonzepts zu machen. Solche positiven Beispiele gibt es bislang noch nicht viele, aber dabei muss es ja nicht bleiben. 
Backes-Gellner, U./Moog, P. (Hrsg.) (2001): Bildungssystem und betriebliche Beschäftigungsstrategien, Beiträge der Jubiläumstagung "25 Jahre Bildungsökonomischer Ausschuss", Schriften des Vereins für Socialpolitik, Bd. 279, Berlin

Baethge, M./Baethge-Kinsky, V./Holm, R./Tullies, K. (2003): Anforderungen und Probleme beruflicher und betrieblicher Weiterbildung. Expertise im Auftrag der Hans-Böckler-Stiftung, Arbeitspapier (76), Düsseldorf Bahnmüller, R. (2009): Qualifizierungstarifverträge - ein Fuß in der Tür, in: Denk-doch-mal.de (1), hrsg. vom Netzwerk für Gesellschaftsethik e.V., http://www.denk-doch-mal.de/node/146 Bahnmüller, R. (2010): Dezentralisierung der Tarifpolitik - ReStabilisierung des Tarifsystems?, in: Bispinck, R./Schulten, T. (Hrsg.): Zukunft der Tarifautonomie. 60 Jahre Tarifvertragsgesetz: Bilanz und Ausblick, Hamburg, S. 81-113

Bahnmüller, R./Bispinck, R./Schmidt, W. (1993): Betriebliche Weiterbildung und Tarifvertrag. Eine Studie über Probleme qualitativer Tarifpolitik in der Metallindustrie, München/Mering

Bahnmüller, R./Fischbach, S. (2006): Qualifizierung und Tarifvertrag. Befunde aus der Metallindustrie Baden-Württembergs, Hamburg Bundesministerium für Bildung und Forschung (BMBF) (2008): Innovationskreis Weiterbildung verabschiedet Empfehlungen, Pressemitteilung 044 vom 5.03.

Breisig, T. (2001): Personalbeurteilung - Mitarbeitergespräch Zielvereinbarungen. Grundlagen, Gestaltungsmöglichkeiten und Umsetzung in Betriebs- und Dienstvereinbarungen, Frankfurt a. M.

Bogumil, J./Grohs, S./Kuhlmann, S./Ohm, A. (2007): Zehn Jahre Neues Steuerungsmodell. Eine Bilanz kommunaler Verwaltungsmodernisierung, Berlin

Busse, G./Seifert, H. (2009): Tarifliche und betriebliche Regelungen zur beruflichen Weiterbildung. Eine explorative Studie, Gutachten für das Ministerium für Bildung und Forschung, Edition der Hans-BöcklerStiftung (233), Düsseldorf

Dobischat, R./Seifert, H. (2001): Betriebliche Weiterbildung und Arbeitszeitkonten, in: WSI-Mitteilungen 54 (2) , S. 92-101

Düll, H./Bellmann, L. (1998): Betriebliche Weiterbildung in Westund Ostdeutschland. Eine theoretische und empirische Analyse mit den Daten des IAB-Betriebspanel 1997, in: Mitteilungen aus der Arbeitsmarkt- und Berufsforschung (MittAB) 39 (2), S. 205-225

Ellguth, P./Kohaut, S. (2011): Der Staat als Arbeitgeber: Wie unterscheiden sich die Arbeitsbedingungen zwischen öffentlichem Sektor und Privatwirtschaft?, in: Industrielle Beziehungen 18 (1-2), S. 11-38

Grass, B. (1997): Die Rolle der Betriebsräte in der Aus- und Weiterbildung, in: Zeitschrift für Personalforschung 11 (2), S. 140-160 Heidemann, W. (2010): Betriebliche Weiterbildung: Tarifverträge und Betriebsvereinbarungen, in: Praxishandbuch WeiterbildungsRecht, Stand: Juni 2010, Seite 3-38

Heimann, K. (1992): Gewerkschaftliche Reformpolitik in einer Qualifikationsgesellschaft. Kritische Zwischenbilanz und Perspektiven im Feld der beruflichen Weiterbildung, in: WSI-Mitteilungen 45 (6), S. 321-329 Johansen, L.-H. (2002): Transferable Training as a Collective Good, in: European Sociological Review 18 (3), S. 301-314

Keller, B. (2010): Arbeitspolitik im öffentlichen Dienst. Ein Überblick über die Arbeitsmärkte und Arbeitsbeziehungen, Modernisierung des öffentlichen Sektors, Sonderband 36, Berlin
Kommission der Europäischen Gemeinschaft (2010): Memorandum über Lebenslanges Lernen, Arbeitsdokument der Kommissionsdienststellen, 30.10., Brüssel

Kreft, J. (2008): Gewerkschaften und Spitzenverbände der Wirtschaft als bildungspolitische Akteure. Positionen, Strategien und Allianzen, Wiesbaden

Kuhlmann, S./Wegrich, K. (2001): Kommunalverwaltung in den neuen Bundesländern: Umbruch, Wandel und Leistungsfähigkeit der lokalen Ebene, in: Schröter, E. (Hrsg.): Empirische Policy- und Verwaltungsforschung: Lokale, nationale und internationale Perspektiven, Opladen, S. $243-260$

Lenz, K./Voß, A. (2009): Analyse der Praxiserfahrungen zum Qualifizierungstarifvertrag der M+E-Industrie NRW. Explorative Kurzstudie, Arbeitspapier (172), hrsg. von der Hans-Böckler-Stiftung, Düsseldorf Leuven, E. (2005): The Economics of Private Sector Training: A Survey of the Literature, in: Journal of Economic Surveys 19 (1), S. 91-111 Meerkamp, A. (2008): Neue Gestaltung des Tarifrechts im öffentlichen Dienst, in: Bispinck, R. (Hrsg.): Verteilungskämpfe und Modernisierung. Aktuelle Entwicklungen in der Tarifpolitik, Hamburg, S. 109-122

Moraal, D. (2009): Standortbestimmung: Berufliche Weiterbildung in Deutschland im europäischen Vergleich, in: Denk-doch-mal.de (1), hrsg. vom Netzwerk für Gesellschaftsethik e.V., http://www.denk-doch-mal. de/node/146

Müller-Jentsch, W. (1999): Berufsbildung - Eine Arena der industriellen Beziehungen?, in: Harney, K./Tenorth, E.-E. (Hrsg.): Beruf und Berufsbildung, Zeitschrift für Pädagogik (40), Beiheft, Weinheim, S. 233-248 Naschold, F./Bogumil, J. (1998): Modernisierung des Staates. New Public Management und Verwaltungsreform, Opladen

Neuberger, O. (2008): Das Mitarbeitergespräch, Leonberg

Schmidt, W./Müller, A./Trittel, N. (2011a): Leistungsentgelt im öffentlichen Dienst: Intentionen, Wirkungen und Akzeptanz, in: Industrielle Beziehungen 18 (1-2), S. 78-98

Schmidt, W./Müller, A./Trittel, N. (2011b): Der Konflikt um die Tarifreform des öffentlichen Dienstes, Projektbericht für die Hans-BöcklerStiftung, Tübingen

Seitz, B. (1997): Tarifierung von Weiterbildung. Eine Problemanalyse in der deutschen Metallindustrie, Opladen

Sterkel, G./Ganser, P./Wiedemuth, J. (Hrsg.) (2010): Leistungspolitik: neu denken. Erfahrungen - Stellschrauben - Strategien, Hamburg Streeck, W. (1988): Skills and the Limits of Neo-Liberalism: The Enterprise of the Future as a Place of Learning, Discussion Papers FS 188-16, hrsg. vom Wissenschaftszentrum Berlin für Sozialforschung and Instituto Juan March de Estudios Investigationes, Madrid

Vereinigte Dienstleistungsgewerkschaft (ver.di )(Hrsg.) (2008): Personalentwicklung in Kommunen. Das Mitarbeiter-Vorgesetzten-Gespräch als Instrument der Personalentwicklung und Erweiterung durch Zielvereinbarungen, Berlin

Vereinigte Dienstleistungsgewerkschaft (ver.di ) (Hrsg.) (2011): Gute Umsetzung des § 5 TVöD/TV-L, Berlin

Wolter, F./Schiener, J. (2009): Einkommenseffekte beruflicher Weiterbildung. Empirische Analysen auf der Basis des Mikrozensus, in: Kölner Zeitschrift für Soziologie und Sozialpsychologie (KZfSS) 61 (1), S. $90-117$ 